\title{
Influence of Intestinal Indigenous Microbiota on Intrafamilial Infection by Helicobacter pylori in Japan
}

\section{Takako Osaki ${ }^{*}$, Cynthia Zaman', Hideo Yonezawa', Yingsong Lin'2, Masumi Okuda ${ }^{3,4}$, Eriko Nozaki ${ }^{5}$, Fuhito Hojo ${ }^{6}$, Satoshi Kurata', Tomoko Hanawa', Shogo Kikuchi' and Shigeru Kamiya ${ }^{1}$}

\begin{abstract}
${ }^{1}$ Department of Infectious Diseases, Kyorin University School of Medicine, Tokyo, Japan, ${ }^{2}$ Department of Public Health, Aichi Medical University School of Medicine, Aichi, Japan, ${ }^{3}$ Department of Pediatrics, Aichi Medical University School of Medicine, Aichi, Japan, ${ }^{4}$ Department of General Medicine and Community Health Science, Hyogo College of Medicine, Hyogo, Japan, ${ }^{5}$ Core Laboratory for Proteomics and Genomics, Kyorin University School of Medicine, Tokyo, Japan, ${ }^{6}$ Graduate School of Medicine, Institute of Laboratory Animals, Kyorin University, Tokyo, Japan
\end{abstract}

Helicobacter pylori is a causative pathogen of chronic gastritis, gastric ulcer disease, and gastric cancer. Humans are known to be a natural host for $\mathrm{H}$. pylori and tend to acquire the pathogen before the age of 5 years. The infection may then persist lifelong if eradication therapy is not applied. One of the modes of transmission of $\mathrm{H}$. pylori is between family members, and therefore, the presence of infected family members is an important risk factor in children. However, other environmental factors have not been fully analyzed. The present study was performed to clarify whether and to what extent intestinal microbiota affect $H$. pylori intrafamilial infection. The fecal specimens from $H$. pylori-infected infants and $H$. pylori-infected and non-infected family members were collected in cohort studies conducted by Sasayama City, Hyogo Prefecture from 2010 to 2013. In total, 18 fecal DNA from 5 families were analyzed. Samples were amplified using $16 \mathrm{~S}$ rRNA universal primers, and the amplicons were sequenced using the lon PGM system. Principal-coordinate analysis demonstrated that there was no difference in intestinal microbiota between $\mathrm{H}$. pylori-positive and H. pylori-negative groups. In intrafamilial comparison tests, the Manhattan distance of intestinal microbiota between the $H$. pylori-infected infant proband and $H$. pylori-negative mother was nearest in the family with low intestinal microbial diversity. However, in the family with the highest intestinal microbial diversity, the nearest Manhattan distance was shown between the $\mathrm{H}$. pyloriinfected infant proband and $\mathrm{H}$. pylori-infected mother. The results in this study showed that the composition of the intestinal microbiota was very similar between members of the same family, and as such, colonization with organisms highly similar to the infected parent(s) may be a risk factor for $\mathrm{H}$. pylori infection in children.

Keywords: Helicobacter pylori, intrafamilial infection, intestinal microbiota, mother-to-child transmission, betadiversity, Manhattan distance

\section{INTRODUCTION}

Humans are the natural host for Helicobacter pylori and more than half of the world's population are infected with this microorganism. Long-term infection with $H$. pylori significantly increases the risk 
of developing site-specific diseases, such as peptic ulcer disease (1), gastric adenocarcinoma $(2,3)$, and mucosa-associated lymphoid tissue lymphoma $(3,4)$.

Helicobacter pylori is most commonly acquired and colonize the human stomach up to the age of 5 years (5), and infection may persist lifelong if eradication therapy is not applied. In the developing countries where $H$. pylori is endemic in the environment, fecal-oral infection through drinking of water contaminated by $H$. pylori-infected patients' feces is an important infection route (6). On the other hand, in the developed countries where the mode of transmission of $H$. pylori is mainly between family members, the presence of infected family members is an important risk factor in children (7-9). Recently, $H$. pylori prevalence in the younger age groups has decreased in Japan (10), and mother-to-child transmission has become the major infection route detected in Japanese children $(9,11-13)$.

After birth, infants are constantly exposed to person-to-person and environmental contact with microbes, and the development of the indigenous microbiota begins. Family microbiota are shared between parents and infants, which plays an important role in the development of the infant microbiome. Current research suggests that the disorder of microbiota induces the gastrointestinal tract.

In our previous study (8), $H$. pylori infection in children was associated with infection in their mothers or fathers, but not in siblings or grandparents. In addition, we reported on intrafamilial infection with $H$. pylori by mother-to-child or father-to-child transmission in three families using multilocus sequencing type analysis of fecal specimens (8). In the present study, it was thought that the mother- or father-to-child transmission of $H$. pylori was more frequent than sibling-to-sibling transmission in this cohort. We focused on the comparison of microbiota between index children with $H$. pylori infection and siblings without $H$. pylori infection. Moreover, we also investigated the diversity of the microbiota between the index child and infected mother or father and whether this affected the routes of $H$. pylori infection for index children.

\section{MATERIALS AND METHODS}

\section{Subjects}

Our previous Sasayama study from 2010 to 2013 in Sasayama city, Hyogo, Japan was undertaken and detailed in previous reports $(8,10,14)$. For the diagnosis of $H$. pylori infection, a TestMate Pylori Antigen enzyme immunoassay T (Wakamoto Co., Ltd., Kanagawa, Japan) and real-time polymerase chain reaction detection of $H$. pylori DNA by the $16 \mathrm{~S}$ rRNA gene of $H$. pyloritargeted primers were used $(15,16)$.

In this study, we analyzed a total of 18 fecal specimens that were collected from $5 \mathrm{H}$. pylori-infected children and their family members.

A modified protocol of the one used in the Sasayama study was undertaken in accordance with the Declaration of Helsinki with approval from the Ethics Committees of Kyorin University, Tokyo.

\section{Extraction of Fecal DNA}

Fecal specimens were stored at $-80^{\circ} \mathrm{C}$ until use. The DNA from the fecal samples was recovered using the QIAamp DNA Stool kit (Qiagen, Germantown, MD, USA) according to the manufacturer's instructions with some modification (10). Two hundred milligrams of the fecal specimens was weighed in a $2 \mathrm{ml}$ microcentrifuge tube containing 0.3 g glass beads, and $180 \mu$ of ATL lysis buffer solution was added (Qiagen). The suspension was mixed using a vortex mixer followed by bead beating three times for $30 \mathrm{~s}$ at a setting of 4,200 rpm using a bead beater (Yasui Kikai, Tokyo, Japan). In addition, $20 \mu \mathrm{l}$ of proteinase $\mathrm{K}$ was added to the mixture. After heating at $56^{\circ} \mathrm{C}$ for $30 \mathrm{~min}$, the bacterial cells in the samples were treated by bead beating in the same manner. After destruction of bacterial cells, total DNA was collected using a Qiagen column and purified according to the manufacturer's instructions. The DNA was extracted in $200 \mu \mathrm{l}$ of AE buffer (Qiagen) with $400 \mu \mathrm{l}$ of ethanol and $20 \mu \mathrm{l}$ of $3 \mathrm{M}$ sodium acetate and kept at $-20^{\circ} \mathrm{C}$ for $14-16 \mathrm{~h}$ for ethanol precipitation. The precipitated DNA was collected by centrifugation for $20 \mathrm{~min}$ at $20,000 \times g$.

\section{S Metagenomic Analysis}

Each DNA specimen was amplified using the Ion $16 \mathrm{~S}^{\mathrm{TM}}$ Metagenomics Kit (Thermo Fisher Scientific, Bremen, Germany). The amplicons were purified and prepared for the sequencing library by using the Ion Plus Fragment Library Kit (Thermo Fisher Scientific) according to the manufacturer's instructions. The library was sequenced by using the Ion PGM system (Thermo Fisher Scientific) and the Ion PGM Hi-Q sequencing kit following the protocol of the kit $(17,18)$.

The operational taxonomic unit of each sequence was determined by the Ion torrent server with the Greengenes database (19). The minimum alignment value calculated for each aligned read in the analysis was $90.0 \%$ for the coverage between hit and query. The number of unique reads needed for the read to be valid was 10 . To make a genus ID, a percentage identity value of $97.0 \%$ was used, and to identify a species ID, $99.0 \%$ was used. If more than one species or genus were found within $0.2 \%$ difference from each other, they were each reported as a "slash ID". The server system was equipped with QIIME (20) for the analysis of microbiota and Emperor (21) for the visualized beta diversities among sequenced samples.

\section{Statistical Analysis}

Statistical analysis was performed using the software KaleidaGraph (Hulinks Inc., Tokyo, Japan). Mean values were compared among subjects using the Kruskal-Wallis test between all groups. For comparing categorical data, the $\chi^{2}$ test was performed. A probability value ( $p$ value) less than 0.05 was considered statistically significant.

\section{RESULTS}

\section{Helicobacter pylori Infection of Family Members}

The 18 fecal specimens from $5 \mathrm{H}$. pylori-infected children and their family members were tested for microbiota analysis. The 
details are shown in Table 1. In total, five families, containing four $H$. pylori-infected mothers and three $H$. pylori-infected fathers, four $H$. pylori-uninfected siblings, and one each uninfected father and mother, as well as five index children were tested. All five $H$. pylori-infected index children had a $H$. pylori-infected mother and/or father but no $H$. pylori-infected sibling.

\section{Relative Abundance of Phyla in Intestinal Microbiota of Subjects Depending on $H$. pylori Status and Age}

The relative abundance of phyla in the feces of each family member is shown in Figure 1. The dominant phyla of the fecal microbiota were Bacteroidetes (12/18 cases) and Firmicutes (6/18 cases).

TABLE 1 | Helicobacter pylori infection-positive children and their families.

\begin{tabular}{lll}
\hline & H. pylori infection positive & H. pylori infection negative \\
\hline Family 1 & Index child and father & Mother and sibling \\
Family 2 & Index child and mother & Sibling \\
Family 3 & Index child and mother & Father and sibling \\
Family 4 & Index child, father, and mother & \\
Family 5 & Index child, father, and mother & Sibling
\end{tabular}

For the comparative study of intestinal microbiota concerning $H$. pylori infection and age, the subjects were divided into $H$. pylori infected and non-infected, and the subgroups were also divided into parent and child groups. In these four groups of subjects, the abundance of Bacteroidetes or Firmicutes and the ratios of Firmicutes/Bacteroidetes were compared (Figure 2). There were no significant differences in the abundance of both phyla by the Kruskal-Wallis test.

\section{Differences in Relative Abundances of Bacterial Families, Genera, and Species in the Intestinal Microbiota of Subjects}

The average relative abundance of each bacterial family, genus, and species was compared among the four groups. At the family level, abundances of Erysipelotrichaceae, Clostridiaceae, and Ruminococcaceae were higher in the $H$. pylori-negative groups than those in the H. pylori positive groups (Figures $3 \mathrm{~A}-\mathrm{C}$ ). At the genus level, a significantly higher abundance of Parasutterella was detected in $H$. pylori-positive children (Figure 3D). Higher abundances of Ruminococcus and Faecalibacterium were also detected in $H$. pylori-negative parents (Figures 3E,F). At the species level, significantly higher abundance of Parasutterella excrementihominis was detected in $H$. pylori-positive children

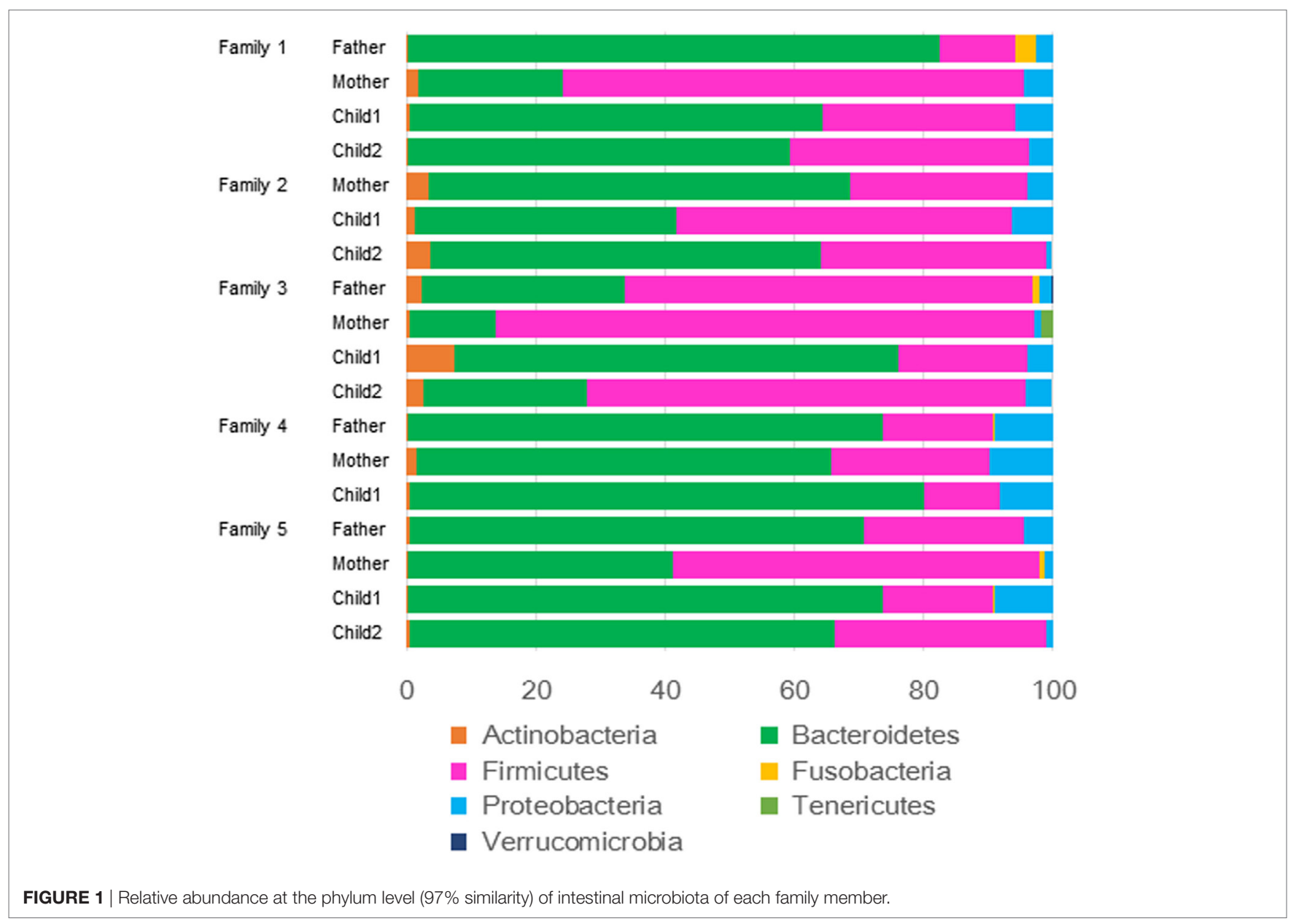


A Bacteroidetes

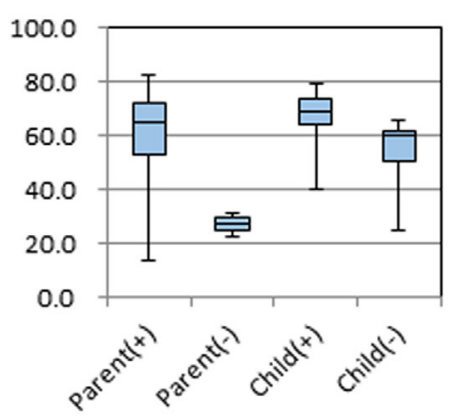

B Firmicutes

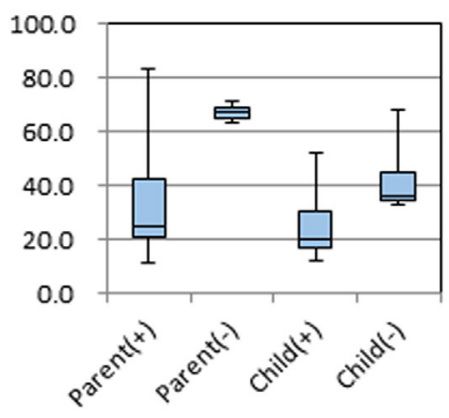

c Firmicutes/Bacteroidetes

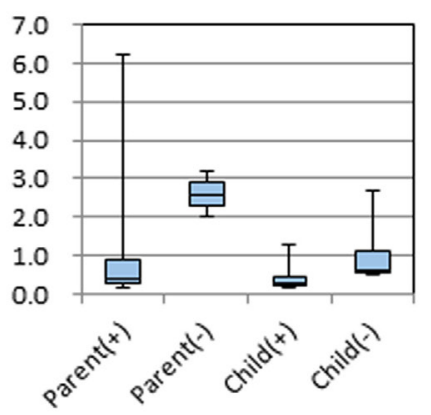

FIGURE 2 | Relative abundances of Bacteroidetes and Firmicutes, and the relative ratio of Firmicutes/Bacteroidetes in microbiota of parents and children in five families with or without Helicobacter pylori infection. (+) and (-) indicate $H$. pylori infection positive and negative, respectively. The middle line in the box plot represents the median value, and the box is drawn from 25 to $75 \%$ quartiles. Whiskers show minimum and maximum values, and the ends of the whiskers represent the non-outlier range.

(Figure 3G). Higher abundances of Faecalibacterium prausnitzii were detected in the microbiota of $H$. pylori-negative parents (Figure 3H), and Clostridium spiroforme was seen in abundance in both $H$. pylori-negative parents and children (Figure 3I). Turicibacter sanguinis was detected only in the microbiota of $H$. pylori-negative parents (two cases). H. pylori was not detected in $H$. pylori-negative and -positive subjects at all from fecal microbiota.

The diversity indices were calculated for the entire sample set according to the $H$. pylori status and age groups. As shown in Figure 4, Simpson's indices were significantly higher in samples from $H$. pylori-negative parents than in those from $H$. pyloripositive parents. These results remained significant even when the comparisons were performed at family and genus levels (Figure 4).

Principal-coordinate analysis (PCoA) of Manhattan distances highlighting similarities between fecal specimens of the 16 family members is shown in Figure 5 and Table 2.

PC1 and PC2 represent the first two highest discriminating axes. At both levels of species and family, family 2 was divided and separated from other families (Figure 5). PCoA plots of other families showed with very near Manhattan distances with exceptional family 1 members, and the beta diversities of the fecal microbiota in families 3,4 , and 5 were close to each other. These results showed that fecal microbiota were similar not only between members of the same family but also between other families.

Manhattan distances at the bacterial family level between family members in each family are shown in Table 2. The Manhattan distances between the index child and other family members show the similarity between the microbiota. Four mothers (underlined in Table 2) and one father (underlined in Table 2) show the highest similarities to the Helicobacter positive index children in the five families. However, one (family 1) of four mothers was $H$. pylori negative, and the other four members were $H$. pylori positive.

In comparing distances from $H$. pylori-positive parent to children in family 1 , the distance between the H. pylori-positive parent to the index child (underlined in Table 2) is shorter than that between mother and sibling (double underlined in Table 2).

\section{DISCUSSION}

Many factors affect the composition of intestinal microbiota (22), and the influence of diet, lifestyle, age, gender, and geography is well known. In this study, it was thought that the background of all subjects was close, since they all lived in the same location (Sasayama city, Japan) and had a similar lifestyle.

The normal human intestinal microbiota comprises two major phyla, the Gram-negative Bacteroidetes and the Gram-positive Firmicutes. In this study, Bacteroidetes existed dominantly in 12 subjects and Firmicutes existed dominantly in other 6 subjects. There were no significant differences in the abundance of dominant phyla between $H$. pylori-positive and -negative groups in children. It is well known that the ratio of Firmicutes/ Bacteroidetes is related to obesity and the body mass index (BMI) of human and animal subjects and that the eradication of $H$. pylori infection induces an increase in BMI $(23,24)$. There have been no previous reports into the ratio of Firmicutes/Bacteroidetes in H. pylori-infected patients. In this study, we compared the ratio between the four groups comprising infected and non-infected children and parents. There was no significant difference in the abovementioned ratio between the four groups. However, in the parents, we only had two $H$. pylori infection-negative subjects. Therefore, the higher ratio of Firmicutes/Bacteroidetes in $H$. pylori-negative parents compared to $H$. pylori-positive parents shown was not significant due to sample size. As a limitation in our study, we were limited by the small sample size, and the number of subjects was very small especially in some subgroups. Additional study using much more samples need to be done in the future.

The average relative abundance numbers for each bacterial family, genus, and species was compared among the four groups. A higher abundance of F. prausnitzii was detected in 


\section{A Erysipelotrichaceae}

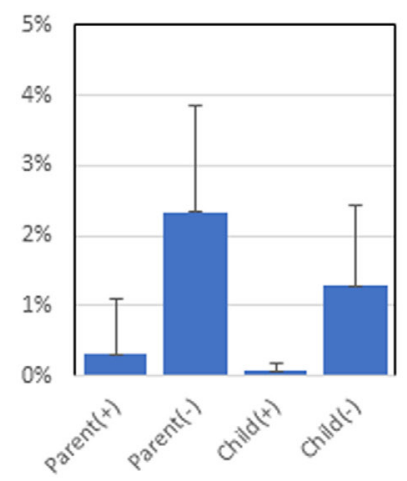

D Parasutterella

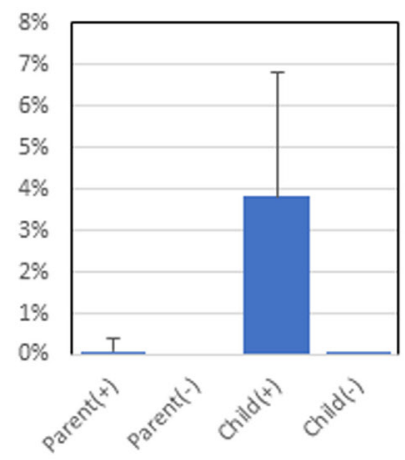

G Parasutterella excrementihominis

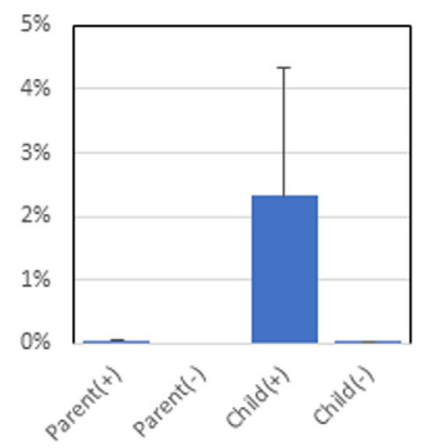

B Ruminococcaceae

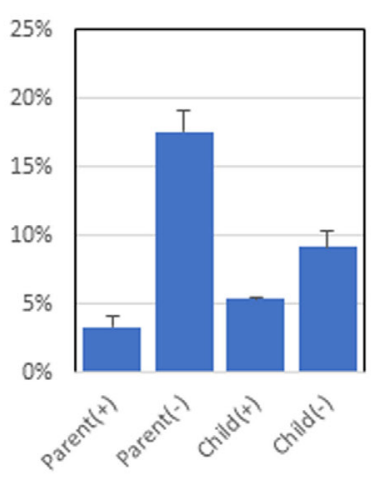

E Ruminococcus

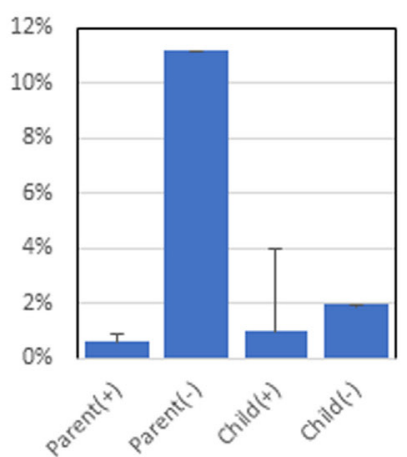

H Faecalibacterium prausnitzii

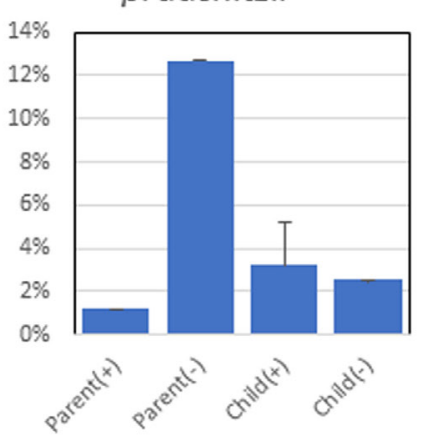

\section{c Clostridiaceae}

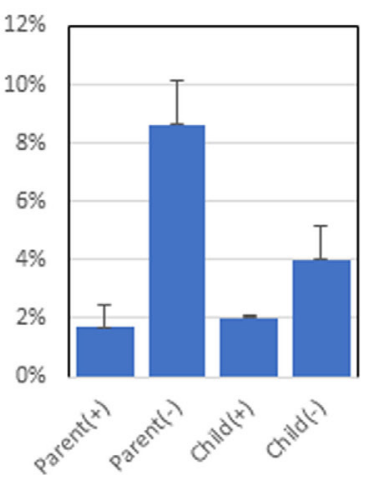

F Faecalibacterium

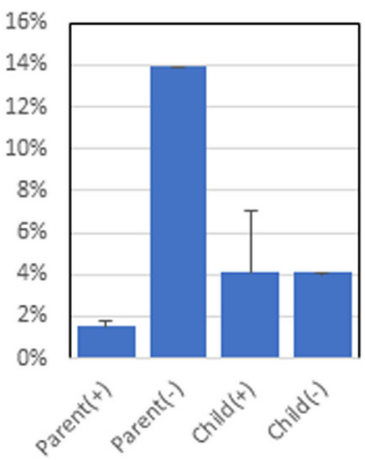

I Clostridium spiroforme

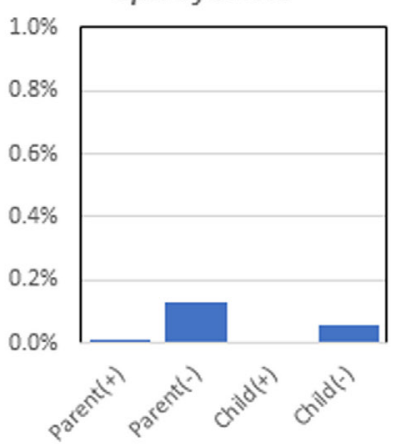

FIGURE 3 | Significant differences (p values < 0.05) in relative abundances of Erysipelotrichaceae (A), Ruminococcaceae (B), Clostridiaceae (C), Parasutterella (D), Ruminococcus (E), Faecalibacterium (F), Parasutterella excrementihominis (G), Faecalibacterium prausnitzii (H), and Clostridium spiroforme (I) composing fecal microbiota. The abundances of the bacteria in bacterial family (A-C), genus (D-F), and species (G-I) were used to determine the statistical significance of differences between groups by the Kruskal-Wallis test.

the microbiota of $H$. pylori-negative parents. F. prausnitzii has been reported to be one of the bacterial species possessing antiinflammatory properties $(25,26)$, and higher abundances of this species were detected preferentially in Japanese subjects with lean body mass (27).

Parasutterella excrementihominis, a member of family Alcaligenaceae, was only detected from the microbiota of H. pylori-positive children. P. excrementihominis was first isolated from the feces of a healthy Japanese male by Nagai et al (28), and they reported that this new species showed more than $98 \% 16 \mathrm{~S}$ rRNA gene sequence similarity to some of the human intestinal uncultured clones reported by several groups in the US and other countries. This indicates that these bacteria are likely to be common members of the human intestinal microbiota. However, we cannot shed more light on why it was detected only in $H$. pylori-positive children in this study, and further studies 


\section{A Species}

\section{Simpson index}

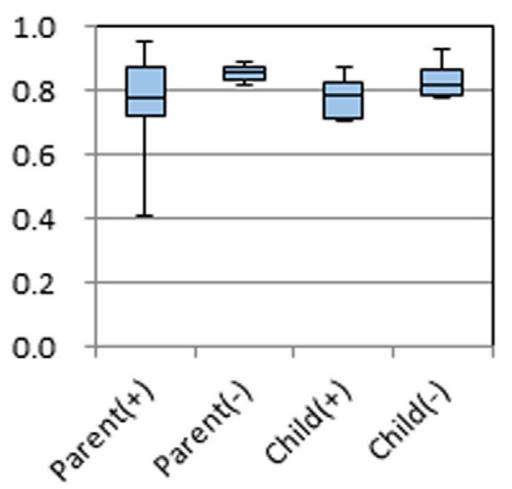

B Genus*

c Family*

FIGURE 4 | Alpha diversity plots of Simpson index measures at species level (A), genus level (B), and family level (C) for the 18 subjects grouped by positive (+) or negative (-) Helicobacter pylori status and by the parent or child in the family. The middle line in the box plot represents the median value, and the box is drawn from 25 to $75 \%$ quartiles. Whiskers show minimum and maximum values, and the ends of the whiskers represent the non-outlier range. $p$-Values of $0.05\left(^{*}\right)$ by the Kruskal-Wallis test were used to determine the statistical significance of differences between groups.

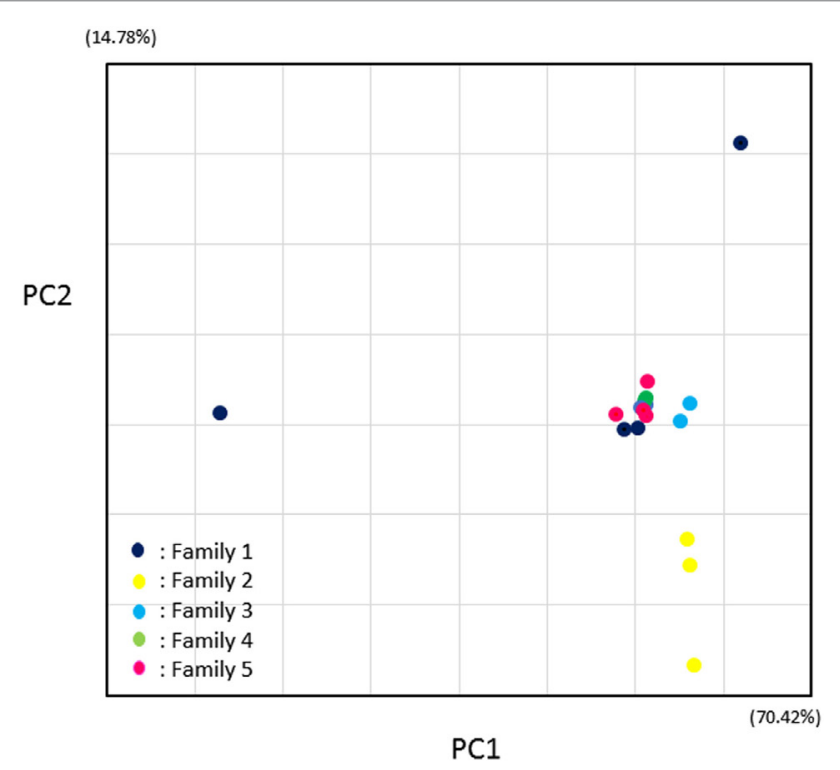

FIGURE 5 | Principal-coordinate analysis of Manhattan distances highlighting differences in intestinal microbiota of family members at the species level. PC1 and PC2 represent the first two highest discriminating axes. The percentage variation explained by each PC axis is indicated. Differently colored symbols represent each family.

are needed to clarify the differences in microbiota between H. pylori-positive and -negative children using a larger subject population.

The average Simpson index values of the four groups were also compared by the Kruskal-Wallis test. Higher average values of Simpson index in the H. pylori-negative groups were detected by comparison at the bacterial genus and family level. These
TABLE 2 | Similarity of microbiota between index child and each family member using Manhattan distance.

\begin{tabular}{|c|c|c|c|c|c|c|}
\hline Family & $\begin{array}{l}\text { H. pylori } \\
\text { infection }\end{array}$ & & $\begin{array}{l}\text { Manhattan } \\
\text { member at }\end{array}$ & $\begin{array}{l}\text { stance of } \\
\text { acterial fan }\end{array}$ & $\begin{array}{l}\text { ach family } \\
\text { illy level }\end{array}$ & \\
\hline Family 1 & $\begin{array}{l}\text { Index child } \\
\text { Father } \\
\text { Mother } \\
\text { Sibling }\end{array}$ & $\begin{array}{l}+ \\
+ \\
(-) \\
(-)\end{array}$ & $\begin{array}{l}\text { Index child } \\
0 \\
308758 \\
\frac{127072}{1207218}\end{array}$ & $\begin{array}{l}\text { Father } \\
0 \\
288172 \\
1496290 \\
\end{array}$ & $\begin{array}{l}\text { Mother } \\
0 \\
1303190\end{array}$ & Sibling \\
\hline Family 2 & $\begin{array}{l}\text { Index child } \\
\text { Mother } \\
\text { Sibling }\end{array}$ & $\begin{array}{l}+ \\
+ \\
(-)\end{array}$ & $\begin{array}{l}\text { Index child } \\
0 \\
\frac{213363}{204327}\end{array}$ & $\begin{array}{l}\text { Mother } \\
0 \\
107814 \\
\end{array}$ & Sibling & \\
\hline Family 3 & $\begin{array}{l}\text { Index child } \\
\text { Father } \\
\text { Mother } \\
\text { Sibling }\end{array}$ & $\begin{array}{l}+ \\
(-) \\
+ \\
(-)\end{array}$ & $\begin{array}{l}\text { Index child } \\
0 \\
178915 \\
\frac{69034}{291835}\end{array}$ & $\begin{array}{l}\text { Father } \\
0 \\
201923 \\
306308\end{array}$ & $\begin{array}{l}\text { Mother } \\
0 \\
\underline{222567} \\
\end{array}$ & Sibling \\
\hline Family 4 & $\begin{array}{l}\text { Index child } \\
\text { Father } \\
\text { Mother }\end{array}$ & $\begin{array}{l}+ \\
+ \\
+\end{array}$ & $\begin{array}{l}\text { Index child } \\
0 \\
51403 \\
\underline{24078} \\
\end{array}$ & $\begin{array}{l}\text { Father } \\
0 \\
60805\end{array}$ & Mother & \\
\hline Family 5 & $\begin{array}{l}\text { Index child } \\
\text { Father } \\
\text { Mother } \\
\text { Sibling }\end{array}$ & $\begin{array}{l}+ \\
+ \\
+ \\
(-)\end{array}$ & $\begin{array}{l}\text { Index child } \\
0 \\
\frac{36008}{92170} \\
84593\end{array}$ & $\begin{array}{l}\text { Father } \\
0 \\
93518 \\
76961 \\
\end{array}$ & $\begin{array}{l}0 \\
49063\end{array}$ & Sibling \\
\hline
\end{tabular}

results showed that the microbiota of $H$. pylori-positive children and adults showed a lower bacterial genus and family diversity than that of $H$. pylori-negative children and parents. In animal studies, $H$. pylori influenced the gastrointestinal microbiota and host immune responses not only locally in the stomach but also distantly as well, affecting important target organs (29). This is 
the first report that gastric infection with $H$. pylori affects the composition of intestinal microbiota in a human host.

Indigenous intestinal microbiota have an ability to protect host organisms against infection by exogenous pathogens (30). However, the role of endogenous intestinal microbiota in the transmission of $H$. pylori is as yet unknown. The origin of $H$. pylori in children is thought to originate from other family members, especially parents. If both the original infected family member and index child are found to have similar microbiota, it is possible that the protective effect by intestinal microbiota on the infection of $H$. pylori may be weak. Microbiota in the intestinal tract of a $H$. pylori-positive person may not have considerable amounts of bacteria that inhibit $H$. pylori. For example, bifidobacteria (31) and lactobacilli differ among individuals, and children inherit it from mother absolutely. Bifidobacteria and lactobacilli are dominant bacteria in infant (32) especially before weaning. It was also reported that Lactobacillus strains have an inhibitory effect against $H$. pylori $(15,33,34)$.

In this study, we also focused on the similarity of the index child and other family members, especially $H$. pylori-positive members and $H$. pylori-negative siblings. In family 1 , the microbiota of the index child was similar to that of the $H$. pylori negative mother; however, in the other four families, lowest Manhattan distances (most similar) were shown between index and $H$. pylori-infected mothers (three cases) and father (only one case). These data showed that the similarity of microbiota was related to transmission of $H$. pylori infection.

The Manhattan distances between $H$. pylori-negative siblings and $H$. pylori-positive parents were compared to those between index children and $H$. pylori-positive parents and shown to be shorter with the exception of family 2 , where all three family members were similar to each other. In family 5 , the $H$. pylori-positive

\section{REFERENCES}

1. Marshall BJ, Barrett LJ, Prakash C, McCallum RW, Guerrant RL. Urea protects Helicobacter (Campylobacter) pylori from the bactericidal effect of acid. Gastroenterology (1990) 99(3):697-702. doi:10.1016/0016-5085(90)90957-3

2. El-Omar EM, Oien K, El-Nujumi A, Gillen D, Wirz A, Dahill S, et al. Helicobacter pylori infection and chronic gastric acid hyposecretion. Gastroenterology (1997) 113:15-24. doi:10.1016/S0016-5085(97)70075-1

3. Fock KM, Talley N, Moayyedi P, Hunt R, Azuma T, Sugano K, et al. AsiaPacific consensus guidelines on gastric cancer prevention. J Gastroenterol Hepatol (2008) 23(3):351-65. doi:10.1111/j.1440-1746.2008.05314.x

4. Nakamura S, Aoyagi K, Furuse M, Suekane H, Matsumoto T, Yao T, et al. B-cell monoclonality precedes the development of gastric MALT lymphoma in Helicobacter pylori-associated chronic gastritis. Am J Pathol (1998) 152(5):1271-9.

5. Weyermann M, Rothenbacher D, Brenner H. Acquisition of Helicobacter pylori infection in early childhood: independent contributions of infected mothers, fathers, and siblings. Am J Gastroenterol (2009) 104(1):182-9. doi:10.1038/ajg.2008.61

6. Nouraie M, Latifi-Navid S, Rezvan H, Radmard AR, Maghsudlu M, ZaerRezaii $\mathrm{H}$, et al. Childhood hygienic practice and family education status determine the prevalence of Helicobacter pylori infection in Iran. Helicobacter (2009) 14(1):40-6. doi:10.1111/j.1523-5378.2009.00657.x

7. Konno M, Yokota S, Suga T, Takahashi M, Sato K, Fujii N. Predominance of mother-to-child transmission of Helicobacter pylori infection detected by random amplified polymorphic DNA fingerprinting analysis in Japanese families. Pediatr Infect Dis J (2008) 27(11):999-1003. doi:10.1097/ INF.0b013e31817d756e index child was similar to the $H$. pylori-positive father, and the $H$. pylori-negative sibling showed a similar microbiome to that of the infected mother implying father-to-child transmission.

In conclusion, our data support the idea that the intestinal microbiota may contribute to intrafamilial transmission of $H$. pylori and that similarity of microbiota could be considered a risk factor. However, it is possible that the similarity in microbiota is also an effect of $H$. pylori infection. This possibility is worthy of further exploration.

\section{ETHICS STATEMENT}

This study was carried out in accordance with the recommendations of ethical guidelines for clinical research in Japan. All experiments were performed in accordance with the principles of Declaration of Helsinki with written informed consents. The study protocol (H22-047-02) was approved by the ethics committees of Kyorin University.

\section{AUTHOR CONTRIBUTIONS}

Conceived and designed the experiments: TO, SKikuchi, SKamiya, and MO. Wrote the paper: TO, CZ, YL, and SKamiya. Metagenome analysis: TO, EN, FH, and HY. Analyzed the data: TO and HY. Contributed reagents/materials/analysis tools: SKurata and TH.

\section{FUNDING}

This project was supported by Grants-in-Aid for Scientific Research (10850148, 15K08471, 15K11426, and 16K08787) from the Japan Society for the Promotion of Science in Japan.

8. Osaki T, Okuda M, Ueda J, Konno M, Yonezawa H, Hojo F, et al. Multilocus sequence typing of DNA from faecal specimens for the analysis of intra-familial transmission of Helicobacter pylori. J Med Microbiol (2013) 62(Pt 5):761-5. doi:10.1099/jmm.0.053140-0

9. Osaki T, Konno M, Yonezawa H, Hojo F, Zaman C, Takahashi M, et al. Analysis of intra-familial transmission of Helicobacter pylori in Japanese families. J Med Microbiol (2015) 64(Pt 1):67-73. doi:10.1099/jmm.0.080507-0

10. Okuda M, Osaki T, Kikuchi S, Ueda J, Lin Y, Yonezawa H, et al. Evaluation of a stool antigen test using a mAb for native catalase for diagnosis of Helicobacter pylori infection in children and adults. J Med Microbiol (2014) 63(Pt 12):1621-5. doi:10.1099/jmm.0.077370-0

11. Yokota S, Konno M, Fujiwara S, Toita N, Takahashi M, Yamamoto S, et al. Intrafamilial, preferentially mother-to-child and intraspousal, Helicobacter pylori infection in Japan determined by mutilocus sequence typing and random amplified polymorphic DNA fingerprinting. Helicobacter (2015) 20(5):334-42. doi:10.1111/hel.12217

12. Yokota S, Toita N, Yamamoto S, Fujii N, Konno M. Positive relationship between a polymorphism in Helicobacter pylori neutrophil-activating protein a gene and iron-deficiency anemia. Helicobacter (2013) 18(2):112-6. doi:10.1111/hel.12011

13. Furuta $Y$, Konno M, Osaki T, Yonezawa H, Ishige T, Imai M, et al. Microevolution of virulence-related genes in Helicobacter pylori familial infection. PLoS One (2015) 10(5):e0127197. doi:10.1371/journal.pone.0127197

14. Okuda M, Osaki T, Lin Y, Yonezawa H, Maekawa K, Kamiya S, et al. Low prevalence and incidence of Helicobacter pylori infection in children: a population-based study in Japan. Helicobacter (2015) 20(2):133-8. doi:10.1111/ hel.12184 
15. Zaman C, Osaki T, Hanawa T, Yonezawa H, Kurata S, Kamiya S. Analysis of the microbial ecology between Helicobacter pylori and the gastric microbiota of Mongolian gerbils. J Med Microbiol (2014) 63(Pt 1):129-37. doi:10.1099/ jmm.0.061135-0

16. Osaki T, Matsuki T, Asahara T, Zaman C, Hanawa T, Yonezawa H, et al. Comparative analysis of gastric bacterial microbiota in Mongolian gerbils after long-term infection with Helicobacter pylori. Microb Pathog (2012) 53(1):12-8. doi:10.1016/j.micpath.2012.03.008

17. Jünemann S, Prior K, Szczepanowski R, Harks I, Ehmke B, Goesmann A, et al. Bacterial community shift in treated periodontitis patients revealed by ion torrent 16S rRNA gene amplicon sequencing. PLoS One (2012) 7(8):e41606. doi:10.1371/journal.pone.0041606

18. Okubo T, Osaki T, Nozaki E, Uemura A, Sakai K, Matushita M, et al. Walker occupancy has an impact on changing airborne bacterial communities in an underground pedestrian space, as small-dust particles increased with raising both temperature and humidity. PLoS One (2017) 12(9):e0184980. doi:10.1371/journal.pone.0184980

19. Edgar RC. Search and clustering orders of magnitude faster than BLAST. Bioinformatics (2010) 26(19):2460-1. doi:10.1093/bioinformatics/btq461

20. Caporaso JG, Kuczynski J, Stombaugh J, Bittinger K, Bushman FD, Costello EK, et al. QIIME allows analysis of high-throughput community sequencing data. Nat Methods (2010) 7(5):335-6. doi:10.1038/nmeth.f.303

21. Vázquez-Baeza Y, Pirrung M, Gonzalez A, Knight R. EMPeror: a tool for visualizing high-throughput microbial community data. Gigascience (2013) 2(1):16. doi:10.1186/2047-217X-2-16

22. Rodríguez JM, Murphy K, Stanton C, Ross RP, Kober OI, Juge N, et al. The composition of the gut microbiota throughout life, with an emphasis on early life. Microb Ecol Health Dis (2015) 26:26050. doi:10.3402/mehd. v26.26050

23. Azuma T, Suto H, Ito Y, Muramatsu A, Ohtani M, Dojo M, et al. Eradication of Helicobacter pylori infection induces an increase in body mass index. Aliment Pharmacol Ther (2002) 16(Suppl 2):240-4. doi:10.1046/j.1365-2036.16.s2.31.x

24. Takeoka A, Tayama J, Yamasaki H, Kobayashi M, Ogawa S, Saigo T, et al. Impact of Helicobacter pylori immunoglobulin G levels and atrophic gastritis status on risk of metabolic syndrome. PLoS One (2016) 11(11):e0166588. doi:10.1371/journal.pone.0166588

25. Carasi P, Racedo SM, Jacquot C, Elie AM, Serradell ML, Urdaci MC. Enterococcus durans EP1 a promising anti-inflammatory probiotic able to stimulate sIgA and to increase Faecalibacterium prausnitzii abundance. Front Immunol (2017) 8:88. doi:10.3389/fimmu.2017.00088

26. Martín R, Miquel S, Benevides L, Bridonneau C, Robert V, Hudault S, et al. Functional characterization of novel Faecalibacterium prausnitzii strains isolated from healthy volunteers: a step forward in the use of F. prausnitzii as a next-generation probiotic. Front Microbiol (2017) 8:1226. doi:10.3389/ fmicb.2017.01226

27. Andoh A, Nishida A, Takahashi K, Inatomi O, Imaeda H, Bamba S, et al. Comparison of the gut microbial community between obese and lean peoples using $16 \mathrm{~S}$ gene sequencing in a Japanese population. J Clin Biochem Nutr (2016) 59(1):65-70. doi:10.3164/jcbn.15-152

28. Nagai F, Morotomi M, Sakon H, Tanaka R. Parasutterella excrementihominis gen. nov., sp. nov., a member of the family Alcaligenaceae isolated from human faeces. Int J Syst Evol Microbiol (2009) 59(Pt 7):1793-7. doi:10.1099/ ijs.0.002519-0

29. Kienesberger S, Cox LM, Livanos A, Zhang XS, Chung J, Perez-Perez GI, et al. Gastric Helicobacter pylori infection affects local and distant microbial populations and host responses. Cell Rep (2016) 14(6):1395-407. doi:10.1016/ j.celrep.2016.01.017

30. Surendran Nair M, Amalaradjou MA, Venkitanarayanan K. Antivirulence properties of probiotics in combating microbial pathogenesis. Adv Appl Microbiol (2017) 98:1-29. doi:10.1016/bs.aambs.2016.12.001

31. Matsuki T, Yahagi K, Mori H, Matsumoto H, Hara T, Tajima S, et al. A key genetic factor for fucosyllactose utilization affects infant gut microbiota development. Nat Commun (2016) 7:11939. doi:10.1038/ncomms11939

32. Bäckhed F, Fraser CM, Ringel Y, Sanders ME, Sartor RB, Sherman PM, et al. Defining a healthy human gut microbiome: current concepts, future directions, and clinical applications. Cell Host Microbe (2012) 12(5):611-22. doi:10.1016/j.chom.2012.10.012

33. Salas-Jara MJ, Sanhueza EA, Retamal-Díaz A, González C, Urrutia H, García A. Probiotic Lactobacillus fermentum UCO-979C biofilm formation on AGS and Caco-2 cells and Helicobacter pylori inhibition. Biofouling (2016) 32(10):1245-57. doi:10.1080/08927014.2016.1249367

34. El-Adawi H, El-Sheekh M, Khalil M, El-Deeb N, Hussein M. Lactic acid bacterial extracts as anti-Helicobacter pylori: a molecular approach. Ir J Med Sci (2013) 182(3):439-52. doi:10.1007/s11845-013-0909-y

Conflict of Interest Statement: The authors declare that the research was conducted in the absence of any commercial or financial relationships that could be construed as a potential conflict of interest.

Copyright (c) 2018 Osaki, Zaman, Yonezawa, Lin, Okuda, Nozaki, Hojo, Kurata, Hanawa, Kikuchi and Kamiya. This is an open-access article distributed under the terms of the Creative Commons Attribution License (CC BY). The use, distribution or reproduction in other forums is permitted, provided the original author(s) and the copyright owner are credited and that the original publication in this journal is cited, in accordance with accepted academic practice. No use, distribution or reproduction is permitted which does not comply with these terms. 